\title{
Guest Editorial: Multimedia Medical Device (MMD) Designing and Clinical Applications
}

\author{
Jiachen Yang ${ }^{1} \cdot$ Zhihan $\mathbf{L v}^{2} \cdot$ Tengfei Yin $^{3}$
}

Published online: 3 September 2016

(C) Springer Science+Business Media New York 2016

Multimedia Medical Device (MMD) is a kind of medical device equipped with multiple sensors, which can capture multimedia information, including video streams, images, voice, heartbeat, blood pressure and scalar sensor data. MMD is able to percept, process in real-time, analyze and evaluate multi-source and multidimensional data, Thus has been widely used in disease diagnosis, rehabilitation, health monitoring, assisted surgery and other medical area. Many of these applications require the multimedia medical sensors paradigm. The notion of ambient intelligent is becoming a reality with the development of variety of multimedia technologies. The intelligence functions of MMD technologies can be applied to Smart Hospital, Smart Clinic, Smart Rehabilitation at Home and so on. Accordingly, the assessment and evaluation methods need to be developed and involved into the iterative designing process.

We have finally selected 16 manuscripts for this special issue after the first, second review processes. Each manuscript selected was blindly reviewed by at least three reviewers consisting of guest editors and external reviewers.

As an important part of traditional medicine, TCM (Traditional Chinese Medicine) has unique and distinct clinical effects in the aspect of disease diagnosis and treatment. Thousands of years of TCM treatment has accumulated abundant clinical data and medical literatures, including valued TCM theories and clinical practice rules. Researchers have conducted various

Zhihan Lv

lvzhihan@gmail.com

Jiachen Yang

Yangjiachen@tju.edu.cn

Tengfei Yin

t.yin@sussex.ac.uk

1 Tianjin University, Tianjin, China

2 SIAT, Chinese Academy of Science, Shenzhen, China

3 Susex University, Brighton, UK 
methods such as clustering analysis, association rules and regression analysis to study TCM theory. However, none of them could reflect well the semantic complexity of TCM and systemic characteristics of TCM treatment. 'TCM clinic records data mining approaches based on weighted-LDA and multirelationship LDA model' conducted a research on the inherent rules of TCM clinic records with a topic model. On the basis of LDA model, weighted mechanism was adopted for each feature word to improve the distinguishing ability and interpretability between the topics. Meanwhile, the modeled topic is taken as the feature for the classification of SVM (Support Vector Machine) to improve the classification accuracy. The experiments showed that analysis of clinical data with topic model can extract TCM treatment rules and provide a novel theoretical method for TCM clinical research.

Overlapping Boundary-Based Multimedia Slice Transcoding Method and Its System for Medical Video and Traffic Video' is concerned about the field of multimedia transcoding technology. It provides a method for multimedia slice transcoding. This method is detailed as follows: the multimedia is segmented according to the preset unit to obtain large numbers of slices; the length of the slices' fuzzy area is acquired; both ends of each slice is made redundant for fuzzy area length; the slices after redundancy are transcoded; the redundant fuzzy area length at both ends of transcoded slice is cut; the slices of cut fuzzy area length are spliced. The method for multimedia slice transcoding stated in this paper can remove the boundary fuzzy area when the transcoded slices are spliced in order to obtain more satisfactory multimedia effects.

'Robot-Assisted Mirror Ultrasound Scanning for Deep Venous Thrombosis Detection Using RGB-D Sensor' proposes a novel "Mirror" robotic US scanning system. The system is composed of two-arm robot, linear US probes for master and slave side, Kinect sensor as a vision servo. 3D images of legs are segmented and register to get mirror matrix. Both Clustered Viewpoint Feature Histogram (CVFH) descriptors of segmented probe and CAD (Computer-Aided Design) training data were calculated to probe recognition. Results from the initial experiment indicate the idea is feasible and promising greatly by improving the inspection efficiently. Clinically, the method can be implemented for pre-operative procedures to predict the risk of DVT, and this may improve the US scanning efficacy.

Health Care and Life Sciences (HCLS) have long been a test-bed for the standards proposed by the W3C to build the Semantic Web. One of the challenges to HCLS Linked Data is access control. 'A Fine-grained Context-aware Access Control Model for Health Care and Life Science Linked Data' presents a fine-grained context-aware access model for HCLS Linked Data based on Semantic Web tools. The model consists of two basic components: ontology base and access policy. Ontology base refers to a set of ontologies that include subject ontology, resource ontology, environment ontology, and action ontology. Results of the analysis indicate that the model expands the scopes of authorization rules for users. Inference of semantic authorization rules is also realized. These rules enable fine-grained access to data and meet the need for dynamic change of HCLS Linked Data.

Anomalous or unnormal multimedia medical devices are to yield anomaly network traffic and affect the diagnosis about medical issues. How to find anomaly network traffic is significantly important for normal applications of multimedia medical devices. 'A Traffic Anomaly Detection Approach in Communication Networks for Applications of Multimedia Medical Devices' studies traffic anomaly detection problem in large-scale communication networks with multimedia medical devices. 
Empirical mode decomposition method and wavelet packet transform are employed to propose an accurate detection method to capture it. Simulation results show that the method is effective and promising.

For many applications of multimedia medical devices in clinical and medical issues, cloud computing becomes a very useful way. However, high energy consumption of cloud computing networks for these applications brings forth a large challenge. 'QoS Constraints-Based Energy-Efficient Model in Cloud Computing Networks for Multimedia Clinical Issues' studies the energy-efficient problem with QoS constraints in large-scale cloud computing networks. Simulation results show that the approach can significantly improve energy efficiency of cloud computing networks.

Wireless multimedia sensor networks are involved with rate-adaptive applications, such as video, audio and images, therefore, high bandwidth or throughput is demanded. To maximize the system throughput of the network, a channel access mechanism based on evolutionary game is proposed in 'An Evolutionary Game Theory-Based Channel Access Mechanism for Wireless Multimedia Sensor Network with Rate-Adaptive Applications'. Under the mechanism, users are assumed to be bounded rationality, in order to coordinate users' behavior in distributed manner, a new reward function is deduced. Theoretical analysis and simulation results show that the reward function proposed can realize the maximization of system throughput and higher normalized utilities of users under the framework of evolutionary game, and the corresponding dynamic equation is globally asymptotically stable, besides, when user deviates because of bounded rationality, the dynamic equation is still able to guarantee faster convergence and smaller performance deviation.

Process innovation is a very great significance for a medical device enterprise to improve its ability to solve problems of lower cost and enhance patients' comfort. 'Study of Medical Device Innovation Design Strategy Based on Demand Analysis and Process Case Base' proposed a concept of process innovation design oriented Web-based process case base system model based on demand mining and conversion. The system conducts demand mining based on product enterprise competitiveness model and features of existence-presentation model, constructs a demand conversion model featuring transition from engineering features to process features, and utilizes browser/sever to erect process case search and exhibition models through realization technique and application flow. It has demonstrated case base backstage realization and management methods, showcased system interface and demonstrated its effectiveness in process design based on actual medical device cases.

In allusion to deficiencies of traditional medical image enhancement algorithms such as poor applicability, large calculated amount and manual parameter setting and local optimum problem of FOA algorithm, 'Study on Medical Image Enhancement Based on IFOA Improved Grayscale Image Adaptive Enhancement' introduces chaos theory into FOA algorithm for improvement based on good global optimum searching performance of fruit fly optimization algorithm and optimizes normalized incomplete Beta function with IFOA for medical image enhancement. The experimental result shows that the improved FOA algorithm can highlight image features effectively, improve visual effect of images and efficiency, avoid invariability of manual parameter adjustment, configure best parameters of normalized incomplete Beta function automatically while guaranteeing best image quality and achieve adaptive enhancement of medical images. 
'Iris Based Secure NROI Multiple Eye Image Watermarking for Teleophthalmology' presents a new secure multiple text and image watermarking scheme on cover eye image using fusion of discrete wavelet transforms (DWT) and singular value decomposition (SVD) for Teleophthalmology. Secure Hash Algorithm (SHA- 512) is used for generating hash corresponding to iris part of the cover digital eye image and this unique hash parameter is used for enhancing the security feature of the proposed watermarking technique. The proposed multilevel watermarking method correctly extracts the embedded watermarks without error and is robust against the all considered attacks without significant degradation of the medical image quality of the watermarked image.

Multi-modal active data such as EEG and physiological signal is increasingly utilized to analyze of human emotional states. Due to the noise existed in collected active data, however, the performance of emotion recognition is still not satisfied. In fact, the issue of emotion recognition can be regarded as channel coding, which focuses on reliable communication through noise channels. Using active data and its label, the redundant codeword would be generated to correct signals noise and recover emotional label information. 'Error-Correcting Output Codes for Multi-label Emotion Classification' utilize multi-label output codes method to improve accuracy and robustness of multi-dimensional emotion recognition by training a redundant codeword model, which is the idea of error-correcting output codes. The experiment results on DEAP dataset show that the multi-label output codes method outperforms other traditional machine learning or pattern recognition methods for the prediction of emotional multi-labels.

The state-of-the-art blind image quality assessment (BIQA) metrics usually require a large amount of human scored images to train a regression model used to judge image quality, which makes the results are heavily dependent on the size of training data. 'Statistical modeling in the shearlet domain for blind image quality assessment' presents an efficient BIQA algorithm based on shearlet transform without using human scored images. This is mainly based on that the degradation of the image leads to significant variation in the spread discontinuities in all directions. However, shearlet transform has a strong ability to localize distributed discontinuities. The natural scene statistics (NSS) of shearlet coefficients are applicable to indicate the variation of image quality. Experimental results on benchmark databases illustrate that the proposed method has a good consistency with the subjective assessment of human beings.

The myriad changes that have accompanied modernization - the 'big data' era, rapid technological advancement, societal transformations on both large and small scales - pose severe challenges to individual, autonomous decision-making behavior, which is vital to the formation of a network public opinion environment. 'Autonomy in Individual Behavior under Multimedia Information' built a model to investigate the impact of cognitive resilience on the individual, autonomous behavior choices. Results indicated that individuals who demonstrate high levels of sensitivity to information and knowledge show relatively strong cognitive resilience.

The performance of traditional routing algorithms are so poor over aspects such as end to end delay, data packet delivery ratio and routing overhead that they cannot provide efficient communication for multi-UAVs carrying out missions synergistically. 'Ant Colony Optimization Based Polymorphism-Aware Routing Algorithm for Ad Hoc UAV Network' is proposed to solve the problems. This algorithm integrates ACO algorithm and dynamic source routing algorithm, the level of pheromone in routes 
which are gained in routing discovery process, is chosen as a standard to choose route and calculated by sensing the distance of a route, the congestion level of a route, and the stability of a route. The simulation results show the APAR algorithm has superiority over traditional algorithms in data package delivery ratio, end to end delay, routing overhead and it is dependable in battlefield environment.

'Outlier Correction Method of Telemetry Data Based on Wavelet Transformation and Wright Criterion of Medical Device' proposed an outlier revision method based on Wright criterion, quadratic difference prediction and wavelet transformation. Making use of wavelet transformation, the original telemetry data is decomposed into multi-level detail and approximation components. In detail component of every level, outliers are distinguished by Wright criterion, and which are corrected by quadratic difference prediction method. After wavelet reconstruction, the revised data will be obtained. This method not only can revise the outliers effectively, but also can reserve the key information in original data. Finally, this method has been proved efficacious and feasible for revising the outliers of telemetry data.

In 'The Foundation of a Color-Chip Evaluation System of Jadeite-Jade Green with Color Difference Control of Medical Device', the commercial evaluation of jadeite-jade color green showed that these levels were sequenced from the top quality fancy vivid green to the last faint green. One-way ANOVA verified the reasonability of the nine levels. Fisher discriminant analysis demonstrated that very high accuracy of $99.21 \%$ was achieved, and only one group from 126 groups was misjudged. Thus, based on the statistical analysis of color parameters in the uniform color space, the color-chip evaluation system of jadeite-jade green with color difference control could be used with high accuracy.

Dr. Jiachen Yang received the M.S. and Ph.D. degrees in Communication and Information Engineering from the Tianjin University, Tianjin, China, in 2005 and 2009, respectively. Currently, he is an associate professor at Tianjin University. In 2014, he was a visiting scholar in the Department of Computer Science, School of Science at Loughborough University, UK. His research interests include stereo camera, stereo vision research, pattern recognition, stereo image displaying and quality evaluation.

Zhihan $\mathbf{L v}$ is an engineer and researcher of virtual/augmented reality and multimedia major in mathematics and computer science, having plenty of work experience on virtual reality and augmented reality projects, engage in application of computer visualization and computer vision. His research application fields widely range from everyday life to traditional research fields (i.e. geography, biology, medicine). During the past years, he has finished several projects successfully on PC, Website, Smartphone and Smartglasses.In 2012, he was granted $\mathrm{PhD}$. degree in Computer applied technology from Ocean university of China (2006-2012). Before that, he has enjoyed sixteen months full-time research experience at Centre national de la recherche scientifique(CNRS)UPR9080 in Paris (2010-2011). After then, he has fulfilled two-year postdoc research experience at Umea university and a short invited teaching experience at KTH Royal Institute of Technology in Sweden. Since 2012, he has held an assistant professor position at Chinese Academy of Science.

Tengfei Yin is presently a Ph.D. researcher in School of Engineering and Informatics at University of Sussex, UK. His current research is in the area of advanced 3D Ultrawideband (UWB) imaging for early breast cancer detection and research articles have been published on IEEE Transactions on Biomedical Engineering and IEEE Antennas and Wireless Propagation Letters. Prior to joining University of Sussex, he received B.Eng. and M.Eng. in Communication Engineering from Qingdao Technological University and Ocean University of China in 2008 and 2011, respectively. His research interests cover microwave image reconstruction, image processing/ machine vision, virtual reality, and peer-to-peer (P2P) network. 